\section{Hofmann, E.}

\section{Erfolgreiches Stressmanagement}

Göttingen, Hogrefe, 2013, 246 Seiten, 22,95 EUR

ISBN 978-3-8017-2490-0

Stress und Stressbewältigung sind seit einigen Jahren vieldiskutierte Themen. In vielen großen und mittelständischen Unternehmen werden hierzu immer mehr Seminare für Mitarbeiter angeboten mit dem Ziel, die Arbeitnehmer vor Stress und den gesundheitlichen Folgen zu schützen und ihnen Methoden zur Stressbewältigung an die Hand zu gegeben. Dennoch beschreibt der Stressreport der Bundesanstalt für Arbeitsschutz und Arbeitsmedizin aus dem Jahre 2012 [Lohmann-Haislah, 2012], dass etwa ein Fünftel der Befragten angibt, vom Arbeitspensum überfordert zu sein, und dass mehr als ein Achtel über fachliche Unterforderung klagt. Diese Statistik wird als wichtiger Indikator für Stresserleben am Arbeitsplatz gewertet. In diesem Zusammenhang wird auch eine Zunahme der körperlichen und psychischen Beschwerden als Folgen von Beanspruchung und Stress berichtet.

Vor diesem Hintergrund steht das Buch «Erfolgreiches Stressmanagement» von Eberhardt Hofmann in einer Reihe von Ratgebern zu dem großen Themenbereich Stress. In den ersten beiden Kapiteln wird dem Leser zum Einstieg eine informative, aber nicht zu ausführliche Einführung in das Stressgeschehen und in die physiologische sowie psychologische Stressreaktion und deren Auswirkungen gegeben. Die in den folgenden 9 Kapiteln dargestellten kognitiv-verhaltenstherapeutisch orientierten Interventionen lassen sich gut aus dieser Einführung ableiten. Als Ansatzpunkte zum Stressabbau stellt Hofmann Atemtechniken zum Abbau von Anspannung, Entspannungsverfahren (auf Basis von Progressiver Muskelrelaxation nach Jacobson) zur muskulären Entspannung, Strategien zur Veränderung von Stressgedanken, grundlegenden Überzeugungen und Persönlichkeitsstilen sowie Maßnahmen zur Prävention und Kompensation des Stressgeschehens mittels Bewegung und Ausdauertraining dar. Zu Letzterem gibt es beispielsweise einen kurzen Abriss über die Trainingslehre und Tipps zum praktischen Trainingsaufbau.

Dem Autor ist es besonders gut gelungen, die verschiedenen Möglichkeiten der kognitiven Umstrukturierung zusammenzutragen, mit Beispielen zu belegen und Hinweise zur praktischen Umsetzung zu geben. Der Fokus liegt dabei nicht nur auf der tatsächlichen Veränderung von Gedanken, sondern auch auf einer emotionalen Neubewertung durch submodale Veränderungen oder Änderungen der Betonung. Um die eigene Persönlichkeit als Stressquelle zu beschreiben, werden zu 7 Verhaltensstilen Teufelskreismodelle dargestellt, die zu einem Stress erzeugenden Zustand führen. Dabei orientiert sich der Autor maßgeblich an den Persönlichkeitsstörungen, wie sie im ICD-10 und DSM-IV beschrieben sind. Zum Stressmanagement werden für jeden Verhaltensstil einzelne Lernziele formuliert, die helfen sollen, aus dem Teufelskreis auszubrechen. Praktische Hilfen zu einer konkreten Umsetzung der Lernziele bleiben jedoch leider aus. Zur Abrundung der kognitiven Strategien stellt der Verfasser zudem Methoden zur Identifikation und Modulierung von Leitideen vor.

Der Autor hat die meisten Elemente des Stressmanagements in das Buch eingearbeitet, die auch andere, vergleichbare Bücher zu diesem Thema aufweisen [z.B. Kaluza, 2012], allerdings sind die Themen größtenteils kompakter dargestellt. Dies hat Vor- und Nachteile. Einerseits wird dadurch ein guter Überblick gegeben, andererseits werden manche Inhalte auf das Nötigste reduziert. Im Buch sind viele praktische Übungen enthalten, die durch Arbeitsblätter angeleitet werden. Dadurch soll sich der Leser nicht nur theoretisch mit der Materie beschäftigen, sondern auch gleich zu konkreten Maßnahmen hinsichtlich des Stressmanagements angeleitet werden. Leider können diese Arbeitsblätter nur im Buch selbst ausgefüllt werden und sind nicht separat (z.B. auf einer $\mathrm{CD})$ verfügbar. Da das Werk so gut wie keine Fachtermini verwendet, richtet es sich zum einen an interessierte Laien, bietet aber auch für Fachleute in Gesundheitswesen und Coaching einen ersten Ansatzpunkt für Interventionen zur Stressbewältigung.

Thilo Rommel, Mainz

\section{Literatur}

Kaluza G: Gelassen und sicher im Stress. Berlin, Springer, 2012.

Lohmann-Haislah A: Stressreport Deutschland 2012. Psychische Anforderungen, Ressourcen und Befinden. Dortmund, Bundesanstalt für Arbeitsschutz und Arbeitsmedizin, 2012 
Petermann, F. (Hrsg.)

\section{Lehrbuch der Klinischen Kinderpsychologie}

Göttingen, Hogrefe, 2013, ed 7, 903 Seiten, 69,95 EUR

ISBN 978-3-8017-2447-4

Die 7. Auflage des «Lehrbuchs der Klinischen Kinderpsychologie» von Franz Petermann und ausgewählten Fachautoren ist als Standardwerk für Studierende der Psychologie und verwandter Disziplinen hervorragend geeignet. Auch für ausgebildete Kinder- und Jugendpsychotherapeuten oder Pädiater mag es als Übersichtswerk noch einiges an Wissenswertem bereithalten.

Das Werk gliedert sich in 45 Kapitel, die 5 großen Abschnitten zugeordnet sind. Im ersten Abschnitt geht es um die Grundlagen der klinischen Kinderpsychologie, wobei auf Begrifflichkeiten und zugrundeliegende Konzepte eingegangen wird. Weiter wird ein Überblick über die Klassifikationssysteme ICD-10 und DSM-IV-TR gegeben, wobei zusätzlich auf das DSM-5 eingegangen wird. Darüber hinaus werden in diesem ersten Abschnitt Entwicklungsaufgaben (Sprachentwicklung, Selbstbehauptung) und mögliche Entwicklungsrisiken von der Geburt bis zum Jugendalter erläutert. Die folgenden 3 Abschnitte befassen sich mit einzelnen Störungsbildern. Dabei wird eine Unterteilung in Entwicklungsstörungen (z.B. Rechenstörung, Enuresis), Verhaltens- und emotionale Störungen (z.B. ADHS, Trennungsangst, selbstverletzendes Verhalten) und körperliche Krankheiten, Suchterkrankungen und andere Beeinträchtigungen der kindlichen Entwicklung (z.B. Adipositas, Kindesmisshandlung) vorgenommen. Die Kapitel zu den jeweiligen Störungen folgen immer dem gleichen Schema: Zu Beginn erfolgt die Beschreibung der Störung, gefolgt von Epidemiologie, Verlauf und möglichen Komorbiditäten. Anschließend werden (ausschließlich verhaltenstherapeutisch geprägte) Erklärungskonzepte vorgestellt. Zum Abschluss folgen Interventionsmöglichkeiten, die je nach Störung recht unterschiedlich detailliert ausfallen. Ein Abschnitt zur Diagnostik findet sich leider nicht für alle Kapitel. Im fünften großen Abschnitt des Lehrbuchs geht es schließlich um verschiedenste Förder- und Therapieansätze, die von Prävention und Frühförderung bis hin zu neuropsychologischer und Psychopharmakotherapie reichen.

Insgesamt ist das Lehrbuch theoretisch und didaktisch sehr gut aufbereitet. So finden sich am Ende eines jeden Kapitels sowohl eine Zusammenfassung und weiterführende Literatur als auch einige Verständnisfragen zum besseren Einprägen und Wiederholen des Gelesenen. Anders als in vorangegangenen Auflagen sind die Antworten auf diese Fragen jedoch nicht mehr im Anhang des Buches aufgeführt, sondern auf einer Webseite. Zur Erleichterung des Verständnisses finden sich in vielen Kapiteln zudem Abbildungen und tabellarische Übersichten. Beispielsweise sind für viele (aber leider wieder nicht für alle) Störungen die Kriterien von ICD-10 und DSMIV-TR tabellarisch gegenübergestellt. Ebenfalls sehr positiv zu bemerken sind die häufig dargestellten Kästen mit dem
Titel «Aktuelle Kontroverse», in denen näher auf aktuelle Entwicklungstrends und widersprüchliche Befunde eingegangen wird. Wer es beim Lesen etwas griffiger und anwendungsbezogener mag und auf Fallbeispiele oder Ähnliches hofft, wird in diesem Lehrbuch jedoch nicht fündig werden. Das Lehrbuch der Klinischen Kinderpsychologie kann allerdings wunderbar als Nachschlagewerk genutzt oder nur zu Teilen gelesen werden. Entsprechend sind einige Definitionen mehrfach in unterschiedlichen Kapiteln vertreten oder bestimmte Begriffe werden häufiger erläutert. Bei kompletter Lektüre dieses Buches könnten diese Redundanzen ein wenig störend wirken.

Etwas undurchsichtig bleibt leider, welche Begriffe mit ausführlichen Erklärungen versehen werden und welche nicht, was das Buch für interessierte Laien nur bedingt empfehlenswert macht. Zudem mag es verwirren, dass manche Erklärungen erst in späteren Kapiteln erfolgen. Außerdem enthalten Querverweise auf ein anderes Kapitel leider weder die entsprechende Referenz noch die Seitenzahl, was das Auffinden des jeweiligen Inhalts unnötig erschwert. Diese der Unabhängigkeit der einzelnen Kapitel geschuldeten Redundanzen und Ungenauigkeiten beeinträchtigen den Lesefluss jedoch eher marginal und fallen insgesamt kaum ins Gewicht.

Zusammenfassend bietet das Lehrbuch viel fachliches Hintergrundwissen und dank dem klar strukturierten Aufbau einen empfehlenswerten Einstieg in die Kinder- (und Jugend-)psychologie. Aufgrund der Informationsvielfalt ist es außerdem hervorragend als Überblick über die aktuellen Standards und Entwicklungen dieses Gebiets geeignet.

Ann-Kathrin Weinke, Mainz 